\title{
Susceptibilidad in vitro de aislamientos colombianos de Plasmodium falciparum a diferentes antipalúdicos
}

\author{
Eliana Arango, Jaime Carmona-Fonseca, Silvia Blair \\ Grupo Malaria, Universidad de Antioquia, Medellín, Colombia
}

Introducción. Los estudios de susceptibilidad in vitro de Plasmodium falciparum a los medicamentos antipalúdicos son herramientas importantes para vigilar la utilidad de esos medicamentos; tales estudios son escasos en Colombia.

Objetivo. Evaluar la susceptibilidad in vitro de aislamientos colombianos de $P$. falciparum a cloroquina, amodiaquina, mefloquina, quinina y artesunato.

Materiales y métodos. Se obtuvieron aislamientos clínicos de $P$. falciparum en varias regiones colombianas. De cada antipalúdico se probaron siete diluciones dobles seriadas, por duplicado, con la técnica HRP-2 y las cepas FCB2 (resistente a cloroquina) y NF54 (sensible a cloroquina) como controles. Se utilizaron las concentraciones inhibitorias $50\left(\mathrm{Cl}_{50}\right)$ mayor de 100, 80, 64, 500 y $10,5 \mathrm{nM}$ como puntos de corte para clasificar la resistencia a cloroquina, amodiaquina, mefloquina, quinina y artesunato, respectivamente.

Resultados. Se evaluaron 25 aislamientos: $72 \%$ de Urabá, $8 \%$ de Bajo Cauca y $20 \%$ de la Costa Pacífica. Las $\mathrm{Cl}_{50}$ promedio de cloroquina, amodiaquina, mefloquina, quinina y artesunato fueron 422,$9 ; 131,4 ; 56,3 ; 269,7$ y $1,88 \mathrm{nM}$, respectivamente, y la proporción de aislamientos resistentes fue, en el mismo orden, $76 \%, 16 \%, 32 \%, 24 \%$ y $4 \%$.

Conclusiones. La baja sensibilidad a la cloroquina concuerda con otros estudios in vitro y con la baja eficacia terapéutica in vivo. Aunque $96 \%$ de los aislamientos fueron sensibles a artesunato, éste y otros estudios han observado aislamientos con disminución de la sensibilidad a las artemisininas $\left(\mathrm{Cl}_{50}>10,5 \mathrm{nM}\right)$, lo que sugiere que el uso indiscriminado de estos medicamentos pone en riesgo su efectividad y podría dejarnos sin opciones para el tratamiento del paludismo por $P$. falciparum.

Palabras clave: malaria/terapia, Plasmodium falciparum, antipalúdicos, resistencia a las drogas, Colombia

In vitro susceptibility of Colombian Plasmodium falciparum isolates to different antimalarial drugs

Introduction. The in vitro assays for susceptibility of Plasmodium falciparum to antimalarial drugs are important tools for monitoring drug resistance, however few such studies have been undertaken in Colombia.

Objective. P. falciparum isolates were obtained from several municipalities in western Colombia (Urabá, Bajo Cauca, Pacific Coast) and characterized for their in vivo susceptibility to chloroquine $(C Q)$, amodiaquine $(A Q)$, mefloquine $(M Q)$, quinine $(Q N)$ and artesunate $(A S)$.

Material and methods. Patients with only $P$. falciparum infection (parasitemia $=1,000$ rings $/ \mu$ l) were included. Each antimalaria drug was tested with 7 dilutions, two-fold doses, with 2 replications and its effect evaluated using the histidine-rich protein (HRP-2) antigen detection method. Controls included FCB2 (chloroquine-resistant) and NF54 (chloroquine-sensitive) strains. $I_{50}>100,80,64,500$ and $10.5 \mathrm{nM}$ were used as the threshold criteria of resistance to $C Q, A Q, M Q, Q N$ and $A S$, respectively.

Results. Twenty-five isolates were evaluated from Urabá (18), Bajo Cauca (2) and the Pacific Coast (5). The mean IC ${ }_{50}$ obtained with $C Q, A Q, M Q, Q N$ and AS were $422.9 ; 131.4 ; 56.3 ; 269.7$ and $1.9 \mathrm{nM}$, respectively, and the number of resistant isolates for these drugs was $19(76 \%), 4$ (16\%), $8(32 \%), 6(24 \%)$ and $1(4 \%)$, respectively. 
Conclusions. The low sensitivity to $C Q$ found here agrees with both in vitro and in vivo studies in Colombia. Ninety-six percent of the isolates were sensitive to AS. However, this study and previous reports have found isolates with low sensitivity to artemisinines $\left(\mathrm{IC}_{50}>10.5 \mathrm{nM}\right)$. This suggests that the indiscriminate use of these drugs put their efficacy at risk and eventually leave no options for falciparum malaria treatment.

Key words: malaria/therapy, Plasmodium falciparum, antimalarials, drug resistance, Colombia

En la mayoría de las regiones con transmisión de paludismo por Plasmodium falciparum se han reportado casos de falla terapéutica y de resistencia a los antipalúdicos de primera línea, como la cloroquina y la sulfadoxina-pirimetamina, ya sea utilizados como monoterapia o en forma combinada $(1,2)$. Con el fin de diseñar esquemas de tratamiento adecuados y de detectar tempranamente cambios en el grado de susceptibilidad, la Organización Mundial de la Salud (OMS) recomienda vigilar regularmente en cada zona endémica la falla terapéutica y la resistencia a los medicamentos antipalúdicos. Para ello, actualmente se usan las pruebas de eficacia terapéutica o pruebas in vivo y las pruebas de susceptibilidad in vitro (2). Las pruebas de susceptibilidad in vitro permiten hacer la caracterización fenotípica de los parásitos como sensibles o resistentes a los antipalúdicos, sin la influencia de los factores de confusión relacionados con el huésped, tales como el grado de inmunidad y la farmacocinética del medicamento (3).

En el 2005 se registraron oficialmente en Colombia 36.190 casos de paludismo por $P$. falciparum (4). Desde 1998 se ha evaluado con los protocolos de la OMS (1998 y 2001) (5,6), la eficacia terapéutica de varios esquemas de tratamiento para el paludismo por $P$. falciparum sin complicaciones; dichos estudios se han centrado en Antioquia y la Costa Pacífica. Por el contrario, han sido pocos y aislados los estudios que han evaluado la susceptibilidad in vitro.

Entre el 2000 y el 2006 el Grupo Malaria de la Universidad de Antioquia evaluó en varios

\footnotetext{
Correspondencia:

Silvia Blair, Calle 62 № 52-59, torre 1, laboratorio 610, Sede de Investigación Universitaria, Universidad de Antioquia, Medellín, Colombia.

Telefax: 2106487

sblair@quimbaya.udea.edu.co

Recibido: 02/11/06; aceptado: 14/05/07
}

municipios antioqueños, la eficacia terapéutica de ocho esquemas de tratamiento antipalúdico y encontró falla de las monoterapias con cloroquina, amodiaquina, mefloquina y sulfadoxinapirimetamina, de $82 \%, 30 \%, 4 \%$ y $24 \%$, respectivamente, y de las terapias combinadas, con cloroquinasulfadoxina-pirimetamina, amodiaquina-sulfadoxinapirimetamina, mefloquina-sulfadoxina-pirimetamina y artesunato-sulfadoxina-pirimetamina, de 17\%, $2 \%, 0 \%$ y $3 \%$, respectivamente (7).

Del lapso entre 1982 y 2003 conocemos seis estudios de susceptibilidad in vitro en Colombia (8-13); los seis evaluaron la cloroquina e informaron resistencia entre $0 \%$ y $97 \%$ (promedio: $59 \%$; intervalo de confianza del $95 \%$ (IC): 49\%$70 \%$ ); cuatro evaluaron la amodiaquina y la resistencia reportada varió entre $0 \%$ y $35 \%$ (promedio: 15\%; IC: 10\%-21\%) (10-13); dos evaluaron la mefloquina (resistencia $8 \%$ y $16 \%$; promedio: $12 \%$; IC: $9 \%-15 \%)(10,13)$; cuatro evaluaron la quinina y sólo en uno se encontró resistencia de $6 \%$, pues en los demás fue cero (8-10,13); finalmente, dos evaluaron la sulfadoxinapirimetamina (resistencia $0 \%$ y $9 \%$; promedio: $5 \%$; IC: $1 \%-8 \%)(11,12)$. Para evaluar la susceptibilidad in vitro de $P$. falciparum a los antipalúdicos, se han desarrollado diferentes métodos que incluyen técnicas microscópicas (método de Rieckmann - Mark III), con radioisótopos (método radioisotópico), inmuno-enzimáticas (método HRP-2 y método DELI) y moleculares, entre otras (14).

El método HRP-2 se basa en la detección de la proteína rica en histidina II de $P$. falciparum, mediante un ensayo inmunoenzimático (ELISA), como una medida del crecimiento de los parásitos; es fácil de ejecutar y no requiere equipos ni material muy especializados, además, tiene sensibilidad similar al método microscópico y es altamente reproducible como el método radioisotópico (15-17). 
El objetivo de este trabajo fue evaluar con el método HRP-2 la susceptibilidad in vitro de los aislamientos colombianos de $P$. falciparum a la cloroquina, la amodiaquina, la mefloquina, la quinina, el artesunato y a los metabolitos monodesetilamodiaquina y dihidroartemisinina. Se utilizaron aislamientos procedentes de cuatro regiones endémicas, ecológica, epidemiológica y socialmente diferentes.

\section{Materiales y métodos}

Medicamentos antipalúdicos. El difosfato de cloroquina, el sulfato de quinina, el diclorhidrato de amodiquina y la dihidroarte-misinina fueron obtenidos de Sigma (Sigma-Aldrich Corp, USA). La monodesetilamodiaquina fue donada por el doctor Pascal Ringwald (Departamento Roll Back Malaria de la OMS), mientras que el clorhidrato de mefloquina y el artesunato fueron donados por laboratorios Mepha (Mepha Ltd., Switzerland).

Predosificación de microplacas. Se utilizaron microplacas de 96 pozos, de fondo plano, marca Falcon (Becton Dickinson Labware, USA). Se evaluaron siete concentraciones por duplicado de cada antipalúdico, preparadas mediante dilución seriada doble, así: cloroquina: 31,3-2002,4 nM; amodiaquina: 34,7-2222,6 nM; monodesetilamodiaquina: 35-2240,9 nM; mefloquina: 7,8-497,3 nM; artesunato: 0,4-24,4 $\mathrm{nM}$ y dihidroartemisinina: 0,5 $33 \mathrm{nM}(17,18)$.

De cada medicamento se preparó una solución madre de $1 \mathrm{mg} / \mathrm{ml}$ con etanol al $70 \%$; luego se prepararon las diluciones seriadas con agua desionizada estéril y se distribuyeron $25 \mu \mathrm{l} /$ pozo de cada una, por duplicado en las microplacas. En los pozos que correspondían a los controles sin tratamiento se distribuyeron $25 \mu \mathrm{l}$ de agua desionizada estéril. Finalmente, las microplacas se dejaron en la cámara de flujo laminar durante toda la noche con el fin de evaporar el solvente y se almacenaron protegidas de la luz y refrigeradas, como máximo, por cinco días (18).

Obtención, preparación y procesamiento de las muestras de sangre. A los pacientes que consultaron en los puestos de diagnóstico de paludismo de los municipios de Turbo y Necoclí (Urabá antioqueño) o en el laboratorio del Grupo
Malaria (Medellín) y a quienes se les diagnosticó infección única por $P$. falciparum, con parasitemia $\geq 1.000$ anillos/ $\mu$ de sangre y que no hubiesen tomado antipalúdicos en las últimas dos semanas, se les informó el objetivo del estudio y, posterior a la aceptación voluntaria de su participación y a la firma del consentimiento informado (aprobado por el Comité de Ética de la Facultad de Medicina de la Universidad de Antioquia), se les diligenció una encuesta y se les tomó una muestra de $5 \mathrm{ml}$ de sangre, por venopunción en el brazo, en tubo con EDTA.

Las muestras se mezclaron con $5 \mathrm{ml}$ de medio incompleto (RPMI 1640 [Sigma] con suplemento de hepes $25 \mathrm{mM}$ [Sigma], hipoxantina 0,2 $\mathrm{mM}$ [Sigma], dextrosa $11 \mathrm{mM}$ [ICN; ICN Biomedicals, Inc, USA], L-glutamina 3,42 mM [Sigma] y gentamicina $16 \mu \mathrm{g} / \mathrm{ml}$ [ICN]) y se refrigeraron a $4{ }^{\circ} \mathrm{C}$ hasta su posterior procesamiento, el cual se realizó durante las 48 horas siguientes a la recolección. Las muestras tomadas en Turbo o Necoclí fueron transportadas refrigeradas hasta el laboratorio en Medellín.

La mezcla sangre-medio se centrifugó a 2.000 rpm durante 10 minutos; se descartaron el plasma, el medio y la capa de glóbulos blancos, y el paquete de glóbulos rojos parasitados se lavó dos veces por centrifugación, utilizando medio incompleto. Luego se preparó una suspensión de glóbulos rojos parasitados con parasitemia de $0,05 \%$ y hematocrito de $1,5 \%$, con glóbulos rojos sanos tipo A+ y medio completo (medio incompleto con suplemento de $\mathrm{NaHCO}_{3} 21,6 \mathrm{mM}$ [J.T. Baker; Mallinckrodt Baker, Inc, USA], glutatión reducido $0,86 \mu \mathrm{g} / \mathrm{ml}[\mathrm{ICN}]$ y $10 \%$ de suero humano inactivado $A_{+}+$). Se distribuyeron $200 \mu$ l por pozo de dicha suspensión en una microplaca predosificada con los antipalúdicos y se incubó durante 72 horas a $37{ }^{\circ} \mathrm{C}$ en atmósfera de $5 \%$ $\mathrm{CO}_{2}, 5 \% \mathrm{O}_{2}$ y $90 \% \mathrm{~N}_{2}$.

Para hacer la dilución de la parasitemia en porcentaje de glóbulos rojos parasitados a partir del recuento de parásitos por microlitro de sangre, se tuvo en cuenta que si un adulto promedio tiene cinco millones de glóbulos rojos/ $/ \mu$ de sangre, una parasitemia con 10.000 anillos/ $\mu$ l equivale a una parasitemia de $0,2 \%$. 
Para realizar los ensayos con las cepas de laboratorio, primero se sincronizaron los cultivos en anillos con la técnica de sorbitol al 5\% (19) y luego se preparó la suspensión de glóbulos rojos parasitados con las mismas condiciones que los aislamientos.

Después de las primeras 24 horas de incubación se preparó un extendido de uno de los pozos control para determinar si había un adecuado crecimiento de los parásitos y al final de las 72 horas de incubación también se determinó la parasitemia de un pozo control; luego las microplacas se congelaron y descongelaron dos veces para obtener una hemólisis completa y se almacenaron $\mathrm{a}-20^{\circ} \mathrm{C}$.

ELISA-HRP2. Para sensibilizar las microplacas de ELISA (microplacas de 96 pozos con fondo en $U$ y de alta fijación [Greiner Bio-One]), el anticuerpo primario MPFM-55A, un anticuerpo monoclonal tipo IgM dirigido contra la proteína HRP-2 y producido por Immunology Consultans Laboratory, Inc, USA (ICL), se diluyó a 1,0 $\mu \mathrm{g} / \mathrm{ml}$ en PBS y se transfirieron $100 \mu \mathrm{l}$ de esta solución a cada pozo. Se incubó a $4{ }^{\circ} \mathrm{C}$ durante toda la noche $\mathrm{y}$, posteriormente, se descartó completamente el contenido de los pozos y se adicionaron $200 \mu \mathrm{l} /$ pozo de solución de bloqueo (PBS-BSA 2\%). Se incubó a temperatura ambiente durante dos horas y luego se descartó completamente el contenido de los pozos y se lavaron las microplacas tres veces con solución de lavado (PBS-Tween-20 0,05\%).

Se descongelaron las microplacas con el cultivo hemolizado y, teniendo en cuenta la parasitemia obtenida en los pozos control después de las 72 horas de incubación, se hizo una dilución de todos los pozos con agua destilada de tal manera que quedara una parasitemia de $0,02 \%$ en el control. De estas diluciones se distribuyeron $100 \mu \mathrm{l} /$ pozo en las microplacas de ELISA sensibilizadas, se incubó por una hora a temperatura ambiente en cámara húmeda y luego se lavó tres veces con solución de lavado. Posteriormente, se diluyó el anticuerpo secundario MPFG-55P (ICL), un anticuerpo monoclonal tipo IgG dirigido contra la proteína HRP-2 y conjugado con peroxidasa, a $0,05 \mu \mathrm{g} / \mathrm{ml}$ en una solución de PBS al $2 \%$, de BSA y $1 \%$ de Tween-20, se transfirieron $100 \mu \mathrm{l}$ de esta solución a cada pozo y se incubó durante una hora a temperatura ambiente en cámara húmeda. Luego se lavó por tres veces y, entonces, se adicionaron $100 \mu \mathrm{l} /$ pozo de 3,3',5,5;tetrametilbenzidina (TMB Single Solution Chromogen/Substrate; Zymed Laboratories, Inc, USA) y se incubó durante 10 minutos a temperatura ambiente en oscuridad. Finalmente, se adicionaron $50 \mu \mathrm{l}$ de solución de parada (ácido sulfúrico $1 \mathrm{M}$ ) y se determinó la densidad óptica (DO) con filtro de $450 \mathrm{~nm}$ en lector de microplacas Bio-Rad 680 (Bio-Rad Laboratories, Inc, USA).

Cultivo continuo de cepas y aislamientos clínicos. Para comparar con el comportamiento de las muestras clínicas, se utilizaron las cepas FCB2 y NF54 como controles. La cepa FCB2 fue aislada y caracterizada en el Instituto Nacional de Salud de Colombia; se obtuvo de un paciente proveniente del municipio de San Martín (Meta) y es resistente a la cloroquina y sensible a la quinina (8). Por su parte, la cepa NF54 fue aislada de un paciente que se encontraba en el aeropuerto de Amsterdam (Holanda) y había estado en África, y es sensible a la cloroquina (20).

Con cada aislamiento también se intentó iniciar un cultivo continuo y para ello se mezclaron 250 $\mu \mathrm{l}$ del paquete de glóbulos rojos parasitados lavados con $250 \mu \mathrm{l}$ de glóbulos rojos sanos y $9,5 \mathrm{ml}$ de medio completo. Tanto las cepas como los aislamientos se mantuvieron con hematocrito de $5 \%$, incubación a $37{ }^{\circ} \mathrm{C}$ en atmósfera de $5 \%$ de $\mathrm{CO}_{2}, 5 \% \mathrm{O}_{2}$ y $90 \% \mathrm{~N}_{2}$, cambio diario de medio y adición de glóbulos rojos sanos dos veces por semana $(19,21)$. Se consideró que los aislamientos estaban adaptados a las condiciones de cultivo in vitro cuando se logró una parasitemia de $2 \%$ o mayor.

\section{Comparación del método HRP-2 con el método} de Rieckmann (Mark III). Con el objetivo de evaluar el desempeño de la prueba HRP-2 frente a un método tradicional, se montaron paralelamente las pruebas HRP-2 y Mark III (22) con las cepas NF54 y FCB2 y con un aislamiento clínico. Los tres cultivos se sincronizaron en estadio de anillo y se diluyeron a una parasitemia de $0,05 \%$ y un hematocrito de $1,5 \%$. Luego se distribuyeron $200 \mu \mathrm{l}$ de dichas diluciones en seis 
microplacas predosificadas con cloroquina, amodiaquina, mefloquina, quinina y artesunato (tres para HRP-2 y tres para Mark III) y se incubaron a $37^{\circ} \mathrm{C}$ en atmósfera de $5 \%$ de $\mathrm{CO}_{2}, 5 \% \mathrm{O}_{2}$ y $90 \% \mathrm{~N}_{2}$. A las 20 y 24 horas posteriores a la incubación se tomaron las tres microplacas Mark III y se descartó el sobrenadante de uno de los pozos control y con el sedimento se preparó una gota gruesa que fue teñida con Giemsa para observar la presencia de esquizontes, y se continuó con la incubación. Cuando se observó que más del $10 \%$ de los parásitos encontrados eran esquizontes, se procedió a finalizar el experimento Mark III. Para la cepa NF54 esto sucedió a las 22 horas, para la FCB2 a las 24 horas y para el aislamiento a las 26 horas.

La finalización del experimento consiste en tomar las microplacas, inclinarlas en un ángulo de $45^{\circ}$, descartar el sobrenadante de cada pozo y preparar una gota gruesa con el sedimento de glóbulos rojos. Dichas gotas gruesas se colorearon con Giemsa y se observaron con objetivo de inmersión. Luego se procedió a determinar la proporción de esquizontes encontrados en un conteo total de 200 parásitos asexuados (22).

Las microplacas HRP-2, por su parte, se incubaron durante 72 horas, se determinó la parasitemia posterior a la incubación de un pozo control y se hemolizaron para luego realizar la prueba de ELISA-HRP-2.

Análisis estadístico. Se construyó una base de datos en Excel $^{\circledR}$, que se analizó con Epilnfo 6,0. Para calcular la $\mathrm{Cl}_{50}$ se usó el programa HN-NonLin que fue diseñado en una hoja de cálculo Excel $^{\circledR}$ y realiza un análisis de regresión no lineal basado en un modelo de regresión polinomial (18).

Para clasificar los aislamientos como sensibles o resistentes a los diferentes antipalúdicos, se tuvieron en cuenta los siguientes puntos de corte de las $\mathrm{Cl}_{50}(23,24)$ : cloroquina: $>100 \mathrm{nM}$; amodiaquina: $>80 \mathrm{nM}$; mefloquina: $>64 \mathrm{nM}$; quinina: $>500 \mathrm{nM}$; artesunato: $>10,5 \mathrm{nM}$ y para los metabolitos monodesetilamodiaquina y dihidroartemisinina se tomaron los puntos de corte correspondientes a amodiaquina y artesunato, respectivamente.

Se calcularon el coeficiente de correlación de Pearson y el coeficiente de determinación para determinar el nivel de correlación entre las $\mathrm{Cl}_{50}$ de todos los tratamientos y entre las prueba HRP-2 y Mark III.

Una probabilidad menor de 0,05 se consideró estadísticamente significativa.

\section{Resultados}

Se evaluaron 25 muestras clínicas, 80\% provinieron del departamento de Antioquia $(72 \%$ de Urabá [18/25] y $8 \%$ del Bajo Cauca [2/25]) y el otro $20 \%$ de la Costa Pacífica colombiana (16\% del departamento del Chocó [4/25] y $4 \%$ del departamento de Nariño [1/25]). Las características generales de los pacientes se presentan en el cuadro 1.

\section{Comparación del ensayo HRP-2 con el método Mark III (visual)}

Las dos cepas y el aislamiento se trabajaron de manera independiente (tres experimentos) y en cada experimento se evaluaron, por Mark III y HRP-2, cinco tratamientos con siete dosis cada uno y dos réplicas de cada dosis. Se encontró una fuerte y significativa correlación entre los resultados obtenidos con ambos métodos $(r=0,897$; $\left.r^{2}=0,805 ; p<0,01\right)$; la concordancia entre los dos métodos para la clasificación de los parásitos como sensibles o resistentes fue del 100\%.

En el cuadro 2 se presentan las $\mathrm{Cl}_{50}$ obtenidas con cada tratamiento para las cepas y el

Cuadro 1. Datos generales de los pacientes.

\begin{tabular}{ll}
\hline Edad (promedio \pm desviación estándar) (años) & $29 \pm 18$ \\
Sexo masculino & $80 \%$ \\
Etnia (mestizos-negros-indígenas) & $80 \%-16 \%-4 \%$ \\
Parasitemia (promedio - intervalo) & 17.868 anillos/ $/ \mu l(1.800-195.520)$ \\
Años de residencia en zona palúdica $(<1 ; 1-5 ;>5)$ & $20 \%-25 \%-55 \%$ \\
Antecedentes de paludismo por P. falciparum en el último año & $35 \%$
\end{tabular}


Cuadro 2. Mark III Vs. HRP-2.

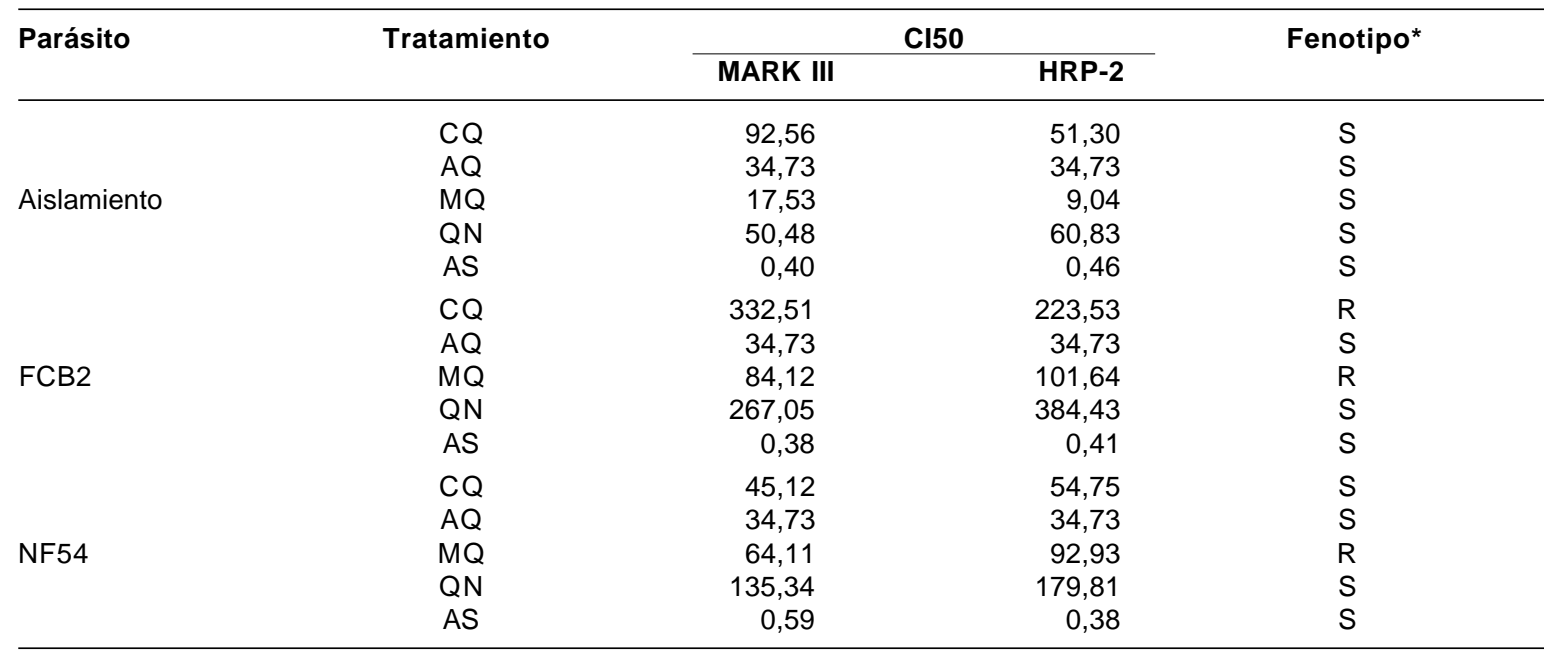

S: sensible, R: resistente. CI50 (nM) máxima para clasificar los parásitos como sensibles a cloroquina (CQ): 100, a amodiaquina (AQ): 80 , a mefloquina (MQ) 64 , a quinina (QN): 500 y a artesunato (AS): $10,5(21,22)$.

aislamiento, y el fenotipo correspondiente. Estos resultados indican que las dos técnicas pueden usarse indistintamente para determinar la susceptibilidad in vitro de $P$. falciparum a los antipalúdicos y comprueban el adecuado desempeño del método HRP-2 que se usó en este estudio.

\section{Susceptibilidad in vitro de las cepas}

Con la misma metodología usada para evaluar los aislamientos, se evaluó la susceptibilidad in vitro de las cepas NF54 (sensible a la cloroquina) y FCB2 (resistente a la cloroquina y sensible a la quinina). Como se muestra en el cuadro 2, tanto con el método visual como con el de HRP-2, se confirmó el fenotipo reportado para cada cepa, lo que confirma el buen desempeño de la metodología aplicada para evaluar la susceptibilidad de los aislamientos. Además, se encontró que aunque, en general las $\mathrm{Cl}_{50}$ de la cepa $\mathrm{FCB} 2$ son mayores que las de la cepa NF54, ambas cepas son sensibles a la amodiaquina, la quinina y el artesunato, y son resistentes a la mefloquina (cuadro 2).

\section{Susceptibilidad in vitro de los aislamientos}

El crecimiento de los 25 aislamientos durante las 72 horas de incubación de la prueba de susceptibilidad fue adecuado. La parasitemia a las 24 horas fue de $0,09 \pm 0,05 \%$ (promedio \pm desviación estándar) y la parasitemia a las 72 horas fue $0,2 \pm 0,09 \%$.

En el cuadro 3 se muestran la $\mathrm{Cl}_{50}$ promedio obtenida para cada antipalúdico y la frecuencia de aislamientos resistentes. En general, las $\mathrm{Cl}_{50}$ encontradas para todos los tratamientos evaluados presentaron un intervalo de variación muy amplio.

Se halló una elevada proporción de $72 \%$ de aislamientos resistentes a la cloroquina, así como otra de $48 \%$ a la amodiaquina o a su metabolito, la monodesetilamodiaquina. La resistencia a estas 4-aminoquinolinas es, por lo tanto, alta. También hubo resistencia de $24 \%$ o más a la quinina y a la mefloquina. Llama la atención la resistencia de $8 \%$ para las artemisininas evaluadas (artesunato y dihidroartemisinina) (un aislamiento con $\mathrm{Cl}_{50}$ de $13,02 \mathrm{nM}$ de artesunato un aislamiento con $\mathrm{Cl}_{50}$ de 14,09 nM de dihidroartemisinina).

Diez y seis por ciento de los aislamientos fueron sensibles a todos los medicamentos; 44\% (11/ 25) fueron resistentes a un solo fármaco (nueve a la cloroquina, uno a la quinina y uno a la monodesetilamodiaquina); $16 \%$ fueron resistentes a dos medicamentos simultáneamente (tres a la cloroquina y la mefloquina y uno a la cloroquina y 
Cuadro 3. Susceptibilidad in vitro de los aislamientos.

\begin{tabular}{lccccc}
\hline Antipalúdico & \multicolumn{3}{c}{ Cl50 (nM) } & Resistentes \\
\cline { 2 - 5 } & Media & IC95\% & Mínimo & Máximo & \\
\hline CQ & 422,94 & $261,88-583,99$ & 29,82 & $1.243,94$ & $19(76 \%)$ \\
AQ & 131,44 & $33,04-229,83$ & 8,38 & $1.043,06$ & $4(16 \%)$ \\
DSA & 169,32 & $66,80-229,83$ & 1,46 & $1.096,29$ & $8(32 \%)$ \\
MQ & 56,28 & $26,81-85,76$ & 2,18 & 348,58 & $8(32 \%)$ \\
QN & 269,71 & $154,43-384,99$ & 8,48 & $1.003,27$ & $6(24 \%)$ \\
AS & 1,88 & $0,71-3,05$ & 0,12 & 13,02 & $1(4 \%)$ \\
DHA & 1,86 & $0,58-3,13$ & 0,32 & 14,09 & $1(4 \%)$ \\
\hline
\end{tabular}

$\mathrm{CQ}$ : cloroquina; $\mathrm{AQ}$ : amodiaquina; DSA: monodesetilamodiaquina; $\mathrm{MQ}$ : mefloquina; $\mathrm{QN}$ : quinina; $\mathrm{AS}$ : artesunato; DHA: dihidroartemisinina

IC: intervalo de confianza

la monodesetilamodiaquina); un solo aislamiento fue resistente a la cloroquina, la monodesetilamodiaquina y la quinina simultáneamente y otro fue resistente a la cloroquina, la monodesetilamodiaquina, la quinina y la mefloquina; $12 \%(3 / 25)$ mostró resistencia simultánea a cinco medicamentos (dos a la cloroquina, la amodiaquina, la monodesetilamodiaquina, la mefloquina y la quinina, y uno a la cloroquina, la amodiaquina, la monodesetilamodiaquina, la mefloquina y la dihidroartemisinina), y un aislamiento fue resistente a todos los fármacos, excepto a la dihidroartemisinina.

\section{Discusión}

Inicialmente se buscó evaluar la susceptibilidad in vitro de los parásitos presentes en muestras criopreservadas (no eran aislamientos frescos) tomadas a pacientes de estudios de eficacia terapéutica realizados entre el 2000 y el 2006 (7). Sin embargo, no fue posible adelantar con éxito el cultivo porque los parásitos requieren más de 20 días para adaptarse y lograr una parasitemia suficiente para realizar las pruebas de susceptibilidad; esta dificultad ya ha sido reportada por otros autores (23-25).

Por esta razón, se decidió trabajar con aislamientos recientes de pacientes procedentes de Urabá y Bajo Cauca antioqueños, de quienes no se conocía la respuesta al tratamiento. Debido a la relativa escasez de enfermos durante el tiempo de ejecución de la investigación, también se incluyeron pacientes provenientes de otros lugares, inclusive fuera de Antioquia.
Aunque el número de aislamientos finalmente evaluados en este estudio es comparable con la cantidad de aislamientos de otros estudios similares realizados en Colombia, es, indudablemente, un número pequeño para establecer el grado de sensibilidad a los medicamentos antipalúdicos, limitación más notoria si se tiene en cuenta que sólo se evaluaron 18 aislamientos de Urabá y dos del Bajo Cauca. No obstante, hay que resaltar que el desarrollo de este estudio permitió la estandarización de la técnica HRP-2, avance que permitirá evaluar un mayor número de aislamientos posteriormente, durante los trabajos futuros.

Los métodos tradicionalmente usados para evaluar la susceptibilidad in vitro de $P$. falciparum a los antipalúdicos son el micrométodo de Rieckmann y el método radioisotópico (14); frente a ellos, la prueba HRP2 es más fácil de ejecutar y tiene sensibilidad similar a la mostrada por los métodos tradicionales (15-17). En el presente estudio, se halló una elevada y significativa correlación entre las $\mathrm{Cl}_{50}$ obtenidas con HRP-2 y el micrométodo de Rieckmann $\left(r=0,897 ; r^{2}=0,805 ; p<0,01\right) y$, además, la clasificación del fenotipo sensible/resistente fue $100 \%$ concordante para todos los tratamientos.

Las cepas FCB2 y NF54, por ser una cloroquinaresistente y la otra cloroquina-sensible, han sido empleadas como controles en estudios, tanto de evaluación de la susceptibilidad in vitro de $P$. falciparum a los antipalúdicos, como de evaluación de la actividad antipalúdica de nuevos compuestos (26-28). La cepa FCB2 fue aislada de un paciente colombiano y fue clasificada como resistente a la 
cloroquina y sensible a la quinina (8); dichos fenotipos fueron confirmados en este estudio y, además, se encontró que es sensible a la amodiaquina, la artesunato y resistente a la mefloquina. En cuanto a la NF54, también se confirmó su fenotipo sensible a la cloroquina característico e, igualmente, mostró sensibilidad a la amodiaquina, la quinina y el artesunato y resistencia a la mefloquina. En general, para todos los tratamientos evaluados, se obtuvieron $\mathrm{Cl}_{50}$ menores con esta última cepa que con la FCB2, lo que sugiere mayor sensibilidad de NF54.

El hecho de haber confirmado en este estudio los fenotipos reportados para cada cepa, valida la metodología utilizada para evaluar la susceptibilidad in vitro de los aislamientos. Como ya se mencionó, el fenotipo frente a la cloroquina de estas dos cepas está muy bien establecido; sin embargo, poco se ha evaluado su comportamiento frente a otros antipalúdicos, lo que hizo difícil comparar estos resultados con otros estudios.

Es importante resaltar que para establecer y validar los puntos de corte para clasificar la resistencia, se deben realizar simultáneamente pruebas de eficacia terapéutica y de susceptibilidad in vitro en una población no inmune (como niños o viajeros) (2). Para la cloroquina, dicho punto de corte fue establecido hace más de 25 años por comparación directa de los resultados obtenidos in vivo e in vitro, mientras que para los demás antipalúdicos, generalmente se han calculado con base solamente en los resultados in vitro, con el promedio más dos desviaciones estándar (29); por lo tanto, no se ha validado su importancia frente a los resultados in vivo, lo cual constituye un factor que explica la falta de concordancia entre las pruebas de eficacia terapéutica y de susceptibilidad in vitro que se ha reportado en los diferentes estudios $(11,12,29$ 31). Todo esto indica que, más importante que conocer la proporción de aislamientos resistentes a un medicamento, es medir la $\mathrm{Cl}_{50}$, lo cual permitirá determinar la sensibilidad basal de los parásitos a un fármaco, evaluar el cambio en dicha sensibilidad en el tiempo y, con estos datos, guiar mejor los estudios de eficacia terapéutica (2).

En 2003, González y colaboradores evaluaron 18 aislamientos de las regiones del Pacífico y amazónica de Colombia y hallaron una frecuencia de resistencia a la cloroquina, la amodiaquina, la mefloquina y la quinina de $78 \%, 35 \%, 17 \%$ y $6 \%$, respectivamente, con el uso de los mismos puntos de corte para la cloroquina y la quinina que se emplearon en este estudio $\left(\mathrm{Cl}_{50}>100 \mathrm{nM}\right.$ para cloroquina y $\mathrm{Cl}_{50}>500 \mathrm{nM}$ para quinina), pero para la amodiaquina y la mefloquina los puntos de corte que utilizaron fueron significativamente menores $\left(\mathrm{Cl}_{50}>20 \mathrm{nM}\right.$ para la amodiaquina, en vez de 80 $\mathrm{nM}$ y $\mathrm{Cl}_{50}>24 \mathrm{nM}$ para la mefloquina, en vez de $64 \mathrm{nM})(13)$.

Dos años después, Aponte y colaboradores evaluaron 24 aislamientos de $P$. falciparum de la región del Pacífico y encontraron una $\mathrm{Cl}_{50}$ promedio a la cloroquina 1,3 veces mayor que la reportada en 2003 y para la mefloquina, la $\mathrm{Cl}_{50}$ promedio hallada en 2005 fue nueve veces mayor que la de 2003 (2003: 10,8 nM; 2005: 97 nM) (13) (Aponte, S. et al. Evaluación de la respuesta in vitro de aislados frescos de Plasmodium falciparum a nuevos medicamentos antipalúdicos. En: Memorias. XII Congreso Colombiano de Parasitología y Medicina Tropical, Bogotá, 2005); lo anterior sugiere una disminución, con el tiempo, de la sensibilidad de los aislamientos colombianos a los antipalúdicos.

En este estudio se observa esa misma tendencia de los aislamientos de $P$. falciparum colombianos a ser cada vez menos sensibles a los antipalúdicos; en efecto, la $\mathrm{Cl}_{50}$ promedio encontrada ahora por nosotros para la cloroquina fue 2,4 veces mayor que la reportada en 2005 , mientras que para la amodiaquina y la quinina fueron 12,8 y 4,5 veces mayores que las de 2003, respectivamente, y en el caso de la mefloquina esta $\mathrm{Cl}_{50}$ fue 5,2 veces mayor que la reportada en 2003, aunque con respecto a la de 2005 fue 1,7 veces menor.

La baja sensibilidad encontrada en este estudio a la cloroquina $(76 \%$ de los aislamientos presentaron $\mathrm{Cl}_{50}>100 \mathrm{nM}$ ) concuerda con los demás estudios de susceptibilidad in vitro en Colombia (8-13), así como con la gran frecuencia de falla terapéutica que ha presentado la monoterapia con la cloroquina en nuestro país y confirma, una vez más, que no debe emplearse en ningún caso la 
monoterapia con este medicamento para el paludismo por $P$. falciparum (7).

La amodiaquina es entre una y tres veces más activa in vitro que su principal metabolito, la monodesetilamodiaquina (32), lo cual fue confirmado en este estudio, al encontrar que la $\mathrm{Cl}_{50}$ promedio para la amodiaquina fue 1,3 veces menor que la $\mathrm{Cl}_{50}$ de la monodesetilamodiaquina, y que cuatro de los 25 aislamientos presentaron $\mathrm{Cl}_{50}$ por encima de $80 \mathrm{nM}$ (punto de corte que los clasifica como resistentes), mientras que para la monodesetilamodiaquina, este punto de corte fue superado por ocho aislamientos.

Estos hallazgos también concuerdan con los estudios que indican que la amodiaquina es efectiva aún en zonas donde la resistencia a la cloroquina es alta $(33,34)$; sin embargo, contrastan con los estudios de eficacia terapéutica de la monoterapia con amodiaquina en Colombia, en donde se reporta una falla de $30 \%$ en Turbo y El Bagre (Antioquia) (7) y de 50\% en Tumaco (Nariño) (35). Esta diferencia entre los resultados obtenidos en estudios in vivo e in vitro con la amodiaquina ya había sido informada en varios estudios. Brasseur y colaboradores en 1995 encontraron, en Camerún y Congo, 20,3\% de falla y sólo $9 \%$ de resistencia (30); por otra parte, Aubouy y colaboradores en 2004 hallaron 40,5\% de falla y 5,4\% de resistencia en Gabón (29), y más recientemente, Tinto y colaboradores, reportaron $30 \%$ de falla y $7 \%$ de resistencia en un estudio en Ruanda (31).

El metabolismo de la amodiaquina en humanos depende de la enzima CYP2C8 y da lugar a la formación de otros metabolitos menores y poco activos, tales como la bidesetilamodiaquina y la hidroxidesetilamodiaquina (32). Además, la enzima CYP2C8 muestra gran variación entre los individuos en el metabolismo de sustratos (36) y presenta diferentes variantes genéticas $(37,38)$. Todos estos factores pueden ayudar también a explicar la falta de concordancia entre los resultados in vivo e in vitro con la amodiaquina.

En un estudio de nuestro grupo de 69 pacientes de tres municipios con paludismo de Colombia (Tumaco, Turbo y El Bagre), se encontró la variante CYP2C ${ }^{\star} 2$ en $6 \%$ de los pacientes y la mutación $\mathrm{R} 139 \mathrm{~K}$ de la variante CYP2C8*3en 3\%; dichas variantes aparecieron principalmente en los pacientes en quienes hubo falla terapéutica de la monoterapia con amodiaquina (39); además, se sabe que la presencia de estas mutaciones reduce el metabolismo del paclitaxel (medicamento anticanceroso) y del ácido araquidónico (38).

Aunque en los estudios de eficacia terapéutica en Antioquia no se incluyó ningún esquema con quinina, este medicamento hace parte de las pautas de tratamiento oficiales de nuestro país, tanto para el paludismo complicado (combinado con sulfadoxina-pirimetamina) como para el esquema de rescate (combinado con clindamicina) para los pacientes que fallan al tratamiento de primera línea (40); en el presente estudio se encontró que la quinina aún inhibe eficazmente el crecimiento de los parásitos, puesto que $76 \%$ de los aislamientos presentaron una $\mathrm{Cl}_{50}$ menor de $500 \mathrm{nM}$.

En lo que se refiere a las artemisininas, en este estudio se halló una $\mathrm{Cl}_{50}$ promedio para el artesunato y la dihidroartemisinina algo menor que la informada en 2003 para arteeter y en 2005 para artesunato en el área del Pacífico colombiano (13) (Aponte, S. et al. Evaluación de la respuesta in vitro de aislados frescos de Plasmodium falciparum a nuevos medicamentos antipalúdicos. En: Memorias. XII Congreso Colombiano de Parasitología y Medicina Tropical, Bogotá, 2005).

Sin embargo, algunos aislamientos manifiestan $\mathrm{Cl}_{50}$ mayores de $10,5 \mathrm{nM}$, tanto en los estudios previos como en el actual y, por lo tanto, presentan una sensibilidad disminuida a estos medicamentos; además, con el esquema artesunato- sulfadoxina-pirimetamina se obtuvo una falla de $3 \%(7)$, todo lo cual sugiere que el uso indiscriminado de las artemisininas pone en riesgo la efectividad de estos medicamentos en nuestro medio, tal como se presentó en Guayana Francesa (41), lo que podría dejarnos sin opciones para el tratamiento del paludismo por $P$. falciparum.

\section{Agradecimientos}

Los autores agradecen a César Segura y Amanda Maestre por la revisión del manuscrito. 


\section{Conflicto de intereses}

Ninguno para declarar.

\section{Financiación}

Este trabajo fue financiado por la Universidad de Antioquia.

\section{Referencias}

1. Bloland P. Drug resistance malaria. Malaria Epidemiology Branch. Centers for Disease Control and Prevention. WHO/CDS/CSR/DRS/2001. Chamblee, GA: World Health Organization; 2001.

2. Ringwald P. Susceptibility of Plasmodium falciparum to antimalarial drugs. Report on global monitoring 19962004. WHO/HTM/MAL/2005.1103. Geneve: World Health Organization; 2005.

3. Kaddouri H, Nakache S, Houzé S, Mentré F, Le Bras J. Assessment of the drug susceptibility of Plasmodium falciparum clinical isolates from Africa by using a Plasmodium lactate dehydrogenase immunodetection assay and an inhibitory maximum effect model for precise measurement of the 50-percent inhibitory concentration. Antimicrob Agents Chemother. 2006;50: 3343-9.

4. Zambrano P. Enfermedades transmitidas por vectores (ETV). Informe final de malaria, semanas 1 a 52 Colombia, 2005. Inf Quinc Epidemiol Nac. 2006;11:49-64.

5. OMS. Evaluación de la eficacia terapéutica de los medicamentos para el tratamiento del paludismo por Plasmodium falciparum sin complicaciones en las Américas. OPS/HCP/HCT/113/98. Washington: Organización Mundial de la Salud; 1998.

6. WHO. Assessment and monitoring of antimalarial drug efficacy for the treatment of uncomplicated falciparum malaria. WHO/HTM/RBM/2003.50. Geneva: World Health Organization; 2003.

7. Blair S, Carmona-Fonseca J, Pineros JG, Ríos A, Álvarez $\mathrm{T}$, Álvarez $\mathrm{G}$, et al. Therapeutic efficacy test in malaria falciparum in Antioquia, Colombia. Malar J. 2006;5:14

8. Espinal C, Moreno E, Guerra P, De la Vega P. Aislamiento y caracterización de cepas colombianas de Plasmodium falciparum. Biomédica. 1982;2:118-28.

9. Arias A, Espinal C, Guerra P, Cortés G. In vitro and in vivo susceptibilities of $P$. falciparum to antimalarial drugs in some regions of Colombia. Acta Med Colomb. 1982;7:385

10. Espinal CA, Cortés GT, Guerra P, Arias AE. Sensitivity of Plasmodium falciparum to antimalarial drugs in Colombia. Am J Trop Med Hyg. 1985;34:675-80.

11. Blair S, Lacharme LL, Fonseca JC, Tobón A. Resistance of Plasmodium falciparum to 3 antimalarials in Turbo (Antioquia, Colombia), 1998. Rev Panam Salud Pública. 2001;9:23-9.

12. Blair S, Lacharme LL, Carmona J, Tobón A. Resistance of $P$. falciparum to antimalarial drugs en Zaragoza (Antioquia, Colombia). Mem Inst Oswaldo Cruz. 2002;97:401-6.

13. González IJ, Varela RE, Murillo C, Ferro BE, Salas J, Giraldo LE, et al. Polymorphisms in cg2 and pfcrt genes and resistance to chloroquine and other antimalarials in vitro in Plasmodium falciparum isolates from Colombia. Trans R Soc Trop Med Hyg. 2003;97: 318-24.

14. Noedl H, Wongsrichanalai C, Wernsdorfer WH. Malaria drug-sensitivity testing: new assays, new perspectives. Trends Parasitol. 2003;19:175-81.

15. NoedI H, Wernsdorfer WH, Miller RS, Wongsrichanalai C. Histidine-rich protein II: a novel approach to malaria drug sensitivity testing. Antimicrob Agents Chemother. 2002;46:1658-64.

16. NoedI H, Wernsdorfer WH, Kollaritsch $\mathbf{H}$, Looareesuwan S, Miller RS, Wongsrichanalai C. Malaria drug-susceptibility testing. HRP-2-based assays: current data, future perspectives. Wien Klin Wochenschr. 2003;115(Suppl.3):23-7.

17. Noedl H, Attlmayr B, Wernsdorfer WH, Kollaritsch H, Miller RS. A histidine-rich protein 2-based malaria drug sensitivity assay for field use. Am J Trop Med Hyg. 2004;71:711-4.

18. NoedI H. Malaria. Department of Immunology and Medicine, Armed Forces Research Institute of Medical Sciences (USAMC-AFRIMS, Bangkok, Thailand) [Consultado: marzo de 2006]. Disponible en: http:// malaria.farch.net.

19. Ljungström I, Perlmann H, Schlichtherle M, Scherf A, Wahlgren $\mathbf{M}$. Methods in malaria research. Manassas, Virginia: Malaria Research and Reference Reagent Resource Center (MR4); 2004

20. Ponnudurai T, Leeuwenberg AD, Meuwissen JH. Chloroquine sensitivity of isolates of Plasmodium falciparum adapted to in vitro culture. Trop Geog Med. 1981;33:50-4.

21. Moreno C. Actividad antiplasmodial in vitro de plantas del género Piper (tesis). Medellín: Universidad de Antioquia; 2005.

22. WHO. In vitro micro-test (Mark III) for the assessment of the response of Plasmodium falciparum to chloroquine, mefloquine, quinine, amodiaquine, sulfadoxine/pyrimethamine and artemisinin. Instructions for use of the in vitro micro-test kit (Mark III). CTC/ MAL/97.20 Rev.2. Geneva: World Health Organization, Division of Control of Tropical Diseases; 2001.

23. Cerutti Junior C, Marques C, Alencar FE, Durlacher RR, Alween A, Segurado AA, et al. Antimalarial drug susceptibility testing of Plasmodium falciparum in Brazil 
using a radioisotope method. Mem Inst Oswaldo Cruz. 1999;94:803-9.

24. Pradines B, Tall A, Rogier C, Spiegel A, Mosnier J, Marrama L, et al. In vitro activities of ferro-chloroquine against 55 Senegalese isolates of Plasmodium falciparum in comparison with those of standard antimalarial drugs. Trop Med Int Health. 2002;7: 265-70.

25. Wongsrichanalai C, Lin K, Pang LW, Faiz MA, Noedl $\mathrm{H}$, Wimonwattrawatee $\mathrm{T}$, et al. In vitro susceptibility of Plasmodium falciparum isolates from Myanmar to antimalarial drugs. Am J Trop Med Hyg. 2001;65:450-5.

26. Rahman NN. Evaluation of the sensitivity in vitro of Plasmodium falciparum and in vivo of Plasmodium chabaudi Malaria to various drugs and their combinations. Med J Malaysia. 1997;52:390-8.

27. Kayser O, Kiderlen AF, Brun R. In vitro activity of aurones against Plasmodium falciparum strains $\mathrm{K} 1$ and NF54. Planta Med. 2001;67:718-21.

28. Garavito G, Rincon J, Arteaga L, Hata Y, Bourdy G, Gimenez A, et al. Antimalarial activity of some Colombian medicinal plants. J Ethnopharmacol. 2006; 107:460-2.

29. Aubouy A, Mayombo J, Keundjian A, Bakary M, Le Bras J, Deloron P. Short report: lack of prediction of amodiaquine efficacy in treating Plasmodium falciparum malaria by in vitro tests. Am J Trop Med Hyg. 2004;71:294-6.

30. Brasseur P, Agnamey P, Ekobo AS, Samba G, Favennec L, Kouamouo J. Sensitivity of Plasmodium falciparum to amodiaquine and chloroquine in central Africa: a comparative study in vivo and in vitro. Trans R Soc Trop Med Hyg. 1995;89:528-30.

31. Tinto H, Rwagacondo C, Karema C, Mupfasoni D, Vandoren W, Rusanganwa E, et al. In-vitro susceptibility of Plasmodium falciparum to monodesethylamodiaquine, dihydroartemisinin and quinine in an area of high chloroquine resistance in Rwanda. Trans R Soc Trop Med Hyg. 2006;100:509-14.

32. Mount DL, Patchen LC, Nguyen-Dinh P, Barber AM, Schwartz IK, Churchill FC. Sensitive analysis of blood for amodiaquine and three metabolites by high- performance liquid chromatography with electrochemical detection. J Chromatogr. 1986;383:375-86.

33. Brasseur P, Guiguemde R, Diallo S, Guiyedi V, Kombila M, Ringwald $\mathbf{P}$, et al. Amodiaquine remains effective for treating uncomplicated malaria in west and central Africa. Trans R Soc Trop Med Hyg. 1999;93: 645-50.

34. Gorissen E, Ashruf G, Lamboo M, Bennebroek J, Gikunda S, Mbaruku G, et al. In vivo efficacy study of amodiaquine and sulfadoxine/ pyrimethamine in Kibwezi, Kenya and Kigoma, Tanzania. Trop Med Int Health. 2000; 5:459-63.

35. Gonzalez I, Padilla J, Giraldo L, Saravia N. Eficacia de amodiaquina y sulfadoxina/pirimetamina en el tratamiento de malaria no complicada por Plasmodium falciparum en Nariño, Colombia, 1999-2002. Biomédica. 2003;23:38-46.

36. Ong CE, Coulter S, Birkett DJ, Bhasker CR, Miners JO. The xenobiotic inhibitor profile of cytochrome P4502C8. Br J Clin Pharmacol. 2000;50:573-80.

37. Dai D, Tang J, Rose R, Hodgson E, Bienstock RJ, Mohrenweiser HW, et al. Identification of variants of CYP3A4 and characterization of their abilities to metabolize testosterone and chlorpyrifos. J Pharmacol Exp Ther. 2001;299:825-31.

38. Dai D, Zeldin DC, Blaisdell JA, Chanas B, Coulter SJ, Ghanayem BI, et al. Polymorphisms in human CYP2C8 decrease metabolism of the anticancer drug paclitaxel and arachidonic acid. Pharmacogenetics. 2001;11:597-607.

39. Guzmán V. Respuesta terapéutica a la mefloquina y la amodiaquina según el CYP450 y el estado nutricional de pacientes con malaria falciparum (tesis). Medellín: Universidad de Antioquia; 2005.

40. Ministerio de Salud. Resolución No. 00412 de 2000. Guía de atención clínica para el diagnóstico y tratamiento de la malaria. Bogotá: Ministerio de Salud: 2000.

41. Jambou R, Legrand E, Niang M, Khim N, Lim P, Volney B, et al. Resistance of Plasmodium falciparum field isolates to in-vitro artemether and point mutations of the SERCA-type PfATPase6. Lancet. 2005;366:1960-3. 